SECTION 31. Economic research, Finance, innovation.

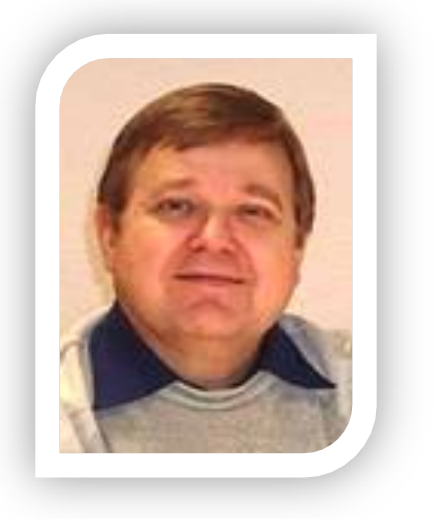

Naumov Anatoly Aleksandrovich candidate of technical Sciences, associate Professor, Center of Applied Mathematical Research, Novosibirsk, Russia

\title{
MATHEMATICAL MODEL OF THE SYSTEM OF CAREER MANAGEMENT OF IT-PRODUCTS OF THE BANK
}

The article describes the mathematical model of the system which allows to recommend the Bank customers to choose the products (services) from the set of products.

Key words: control system, Bank, services.

\section{МАТЕМАТИЧЕСКИЕ МОДЕЛИ СИСТЕМЫ УПРАВЛЕНИЯ ПРОДВИЖЕНИЕМ ІТ-ПРОДУКТОВ БАНКА}

В статье рассмотрены математические модели системы, которая позволяет рекомендовать клиентам банка выбирать продукты (услуги) из множества продуктов.

Ключевые слова: система управления, банк, услуги.

Введение. В работе рассмотрены математические модели системы, которая позволяет рекомендовать клиентам банка выбирать продукты (услуги) из множества продуктов $P$ (см. [1], [2]). Заметим, что в общем случае множество продуктов $P$ может быть не фиксированным. Последнее означает, что продукт может быть построен (синтезирован) на основе методов подбора (выбора, согласования с клиентами) его параметров. Такие продукты, которые будут построены в результате согласования их параметров с клиентами, назовем «гибкими продуктами» или «синтезированными продуктами».

Исходными данными для решения задачи построения такой системы служат: характеристики (параметры) клиентов, статистики по работе клиентов с продуктами из множества $P$ в прошлые периоды времени, критерии оценивания эффективности продуктов для банка и клиента, характеристика (параметры) продуктов. 
Решение задачи. Рассмотрим один из возможных подходов к решению задачи разработки системы продвижения продуктов банка.

Введем обозначения. Пусть $P=\left\{p_{1}, p_{2}, p_{3}, \ldots, p_{n}\right\}$ - множество продуктов (услуг), предоставляемы банком клиентам; $C=\left\{c_{1}, c_{2}, c_{3}, \ldots, c_{l}\right\}$ - множество клиентов банка (в это множество входят клиенты, которые заключали договора с банком $\left(C_{w}\right)$ и не заключали договора $\left.\left(C_{\bar{w}}\right)\right) ; X_{p}-$ множество характеристик продуктов банка $P ; X_{p_{i}}-$ множество характеристик продукта $p_{i} \in P, i=1,2,3, \ldots, n ; \quad X_{c}-$ множество характеристик клиентов банка $C ; X_{c_{i}}-$ множество характеристик клиента $c_{i} \in C, i=1,2,3, \ldots, l ; \quad \vec{Q}=\left\{Q_{1}, Q_{2}, Q_{3}, \ldots, Q_{m}\right\} \quad$ - вектор показателей эффективности, $\vec{Q}_{b}$ - для банка, $\vec{Q}_{c}$ - для клиента.

Сформируем в табличном виде информацию о клиентах и продуктах. В табл. 1 представлена информация о работе клиентов с банком. Эффективности продуктов $p_{i} \in P, i=1,2,3, \ldots, n$, для банка и клиентов оценим и сведем в таблицу (см. табл. 2). Составим таблицу для приоритетных направлений развития (продвижения) продуктов $p_{i} \in P, i=$ $1,2,3, \ldots, n$ банком (см. табл. 3). Аналогично могут быть представлены в табличном виде данные для клиентов, которые консультировались в банке, но договора не заключали (для клиентов из множества $\mathrm{C}_{\overline{\mathrm{w}}}$ ).

Таблица 1.

Информация о клиентах и продуктах.

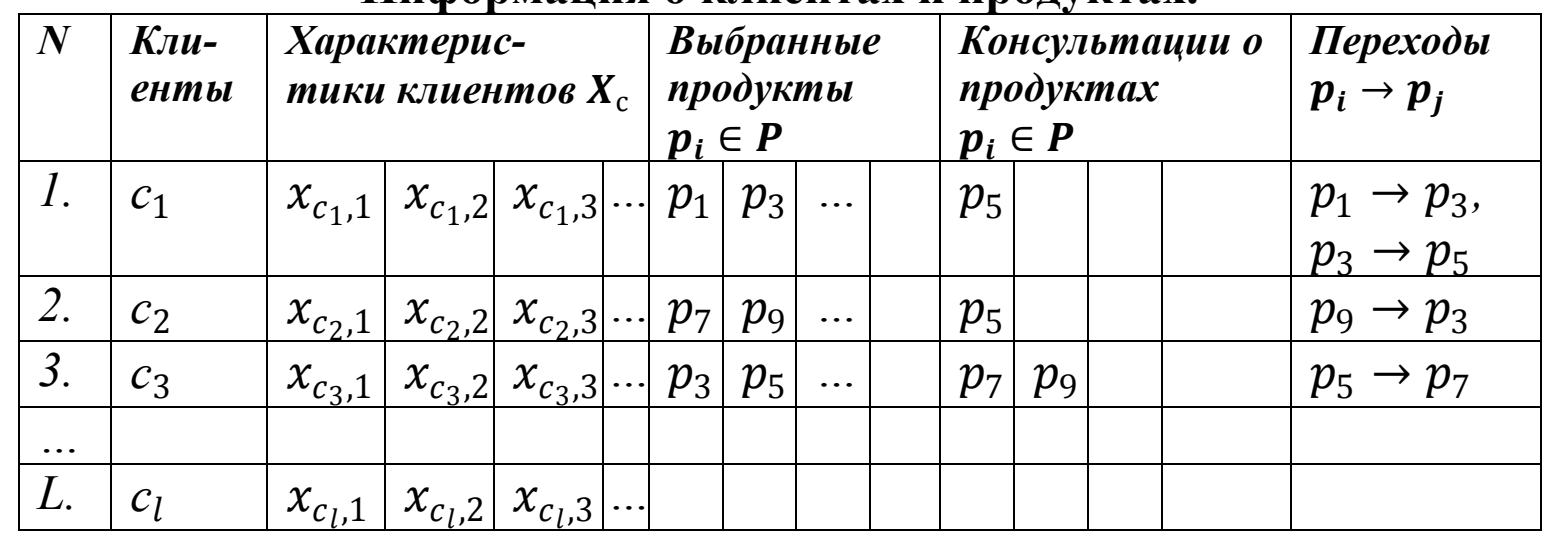

Таблица 2.

Эффективности продуктов для банка и клиентов.

\begin{tabular}{|c|c|c|c|c|c|c|c|c|c|c|}
\hline$N$ & $\begin{array}{l}\text { Продук- } \\
\text { mbl }\end{array}$ & $\begin{array}{l}\text { Xapa } \\
\text { ки } n p\end{array}$ & $\begin{array}{l}\text { lepuc } \\
\text { yкm }\end{array}$ & & $\begin{array}{l}\text { Эффек } \\
\text { для бан }\end{array}$ & $\begin{array}{l}\text { ubHocm } \\
\vec{Q}_{b}\end{array}$ & & $\begin{array}{l}\text { Эффеки } \\
\text { для кли }\end{array}$ & $\begin{array}{l}\text { тивноси } \\
\text { ента } \vec{Q}\end{array}$ & \\
\hline 1. & $p_{1}$ & $x_{p_{1}, 1}$ & $x_{p_{1,2}}$ & $\ldots$ & $Q_{b, p_{1}, 1}$ & $Q_{b, p_{1}, 2}$ & $\ldots$ & $Q_{c, p_{1}, 1}$ & $Q_{c, p_{1}, 2}$ & \\
\hline 2. & $p_{2}$ & $x_{p_{2,1}}$ & $x_{p_{2,2}}$ & $\ldots$ & & & $\cdots$ & & & 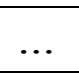 \\
\hline 3. & $p_{3}$ & $x_{p_{3}, 1}$ & $x_{p_{3}, 2}$ & $\ldots$ & $Q_{b, p_{3}, 1}$ & $Q_{b, p_{3}, 2}$ & $\ldots$ & $Q_{c, p_{3}, 1}$ & $Q_{c, p_{3}, 2}$ & $\ldots$ \\
\hline & 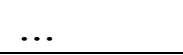 & & & & & & & & & \\
\hline$n$. & $p_{n}$ & $x_{p_{n}, 1}$ & $x_{p_{n}, 2}$ & $\ldots$ & $Q_{b, p_{n}, 1}$ & $Q_{b, p_{n}, 2}$ & $\cdots$ & $Q_{c, p_{n}, 1}$ & $Q_{c, p_{n}, 2}$ & \\
\hline
\end{tabular}


Таблица 3.

Приоритетные направления развития (продвижения) продуктов банком.

\begin{tabular}{|c|c|c|c|c|c|c|c|c|c|}
\hline$N$ & $\begin{array}{l}\text { Продук } \\
-\boldsymbol{m b l} \\
p_{i} \in P\end{array}$ & \multicolumn{4}{|c|}{ План продвижения продуктов $p_{i}$ банком } & \multicolumn{4}{|c|}{$\begin{array}{l}\text { Приоритеты в } \\
\text { соответствии с } \\
\text { критерием } Q_{b, p_{i}, p_{j}} \\
\left(\text { или } Q_{b, p_{i} \rightarrow p_{j}}\right)\end{array}$} \\
\hline 1. & $p_{1}$ & $\begin{array}{l}p_{1} \rightarrow p_{3} \\
Q_{b, p_{1} \rightarrow p_{3}}\end{array}$ & $\begin{array}{l}p_{1} \rightarrow p_{5} \\
Q_{b, p_{1} \rightarrow p_{5}}\end{array}$ & $\begin{array}{l}p_{1} \rightarrow p_{7} \\
Q_{b, p_{1} \rightarrow p_{7}}\end{array}$ & & 0.5 & 0.4 & 0.1 & \\
\hline 2. & $p_{2}$ & $\begin{array}{l}p_{2} \rightarrow p_{4} \\
Q_{b, p_{2} \rightarrow p_{4}}\end{array}$ & $\begin{array}{l}p_{2} \rightarrow p_{8} \\
Q_{b, p_{2} \rightarrow p_{8}}\end{array}$ & & & 0.7 & 0.3 & & \\
\hline 3. & $p_{3}$ & $\begin{array}{l}p_{3} \rightarrow p_{5} \\
Q_{b, p_{3} \rightarrow p_{5}}\end{array}$ & $\begin{array}{l}p_{3} \rightarrow p_{6} \\
Q_{b, p_{3} \rightarrow p_{6}}\end{array}$ & $\begin{array}{l}p_{3} \rightarrow p_{9} \\
Q_{b, p_{3} \rightarrow p_{9}}\end{array}$ & $\ldots$ & 0.6 & 0.2 & 0.1 & $\ldots$ \\
\hline$\ldots$ & & & & & & & & & \\
\hline$n$. & $p_{n}$ & $\begin{array}{l}p_{n} \rightarrow p_{2} \\
Q_{b, p_{n} \rightarrow p_{2}}\end{array}$ & $\begin{array}{l}p_{n} \rightarrow p_{4} \\
Q_{b, p_{n} \rightarrow p_{4}}\end{array}$ & $\begin{array}{l}p_{n} \rightarrow p_{6} \\
Q_{b, p_{n} \rightarrow p_{6}}\end{array}$ & $\ldots$ & 0.3 & 0.2 & 0.15 & ... \\
\hline
\end{tabular}

Представим графически данные о клиентах банка (см. рис. 1).

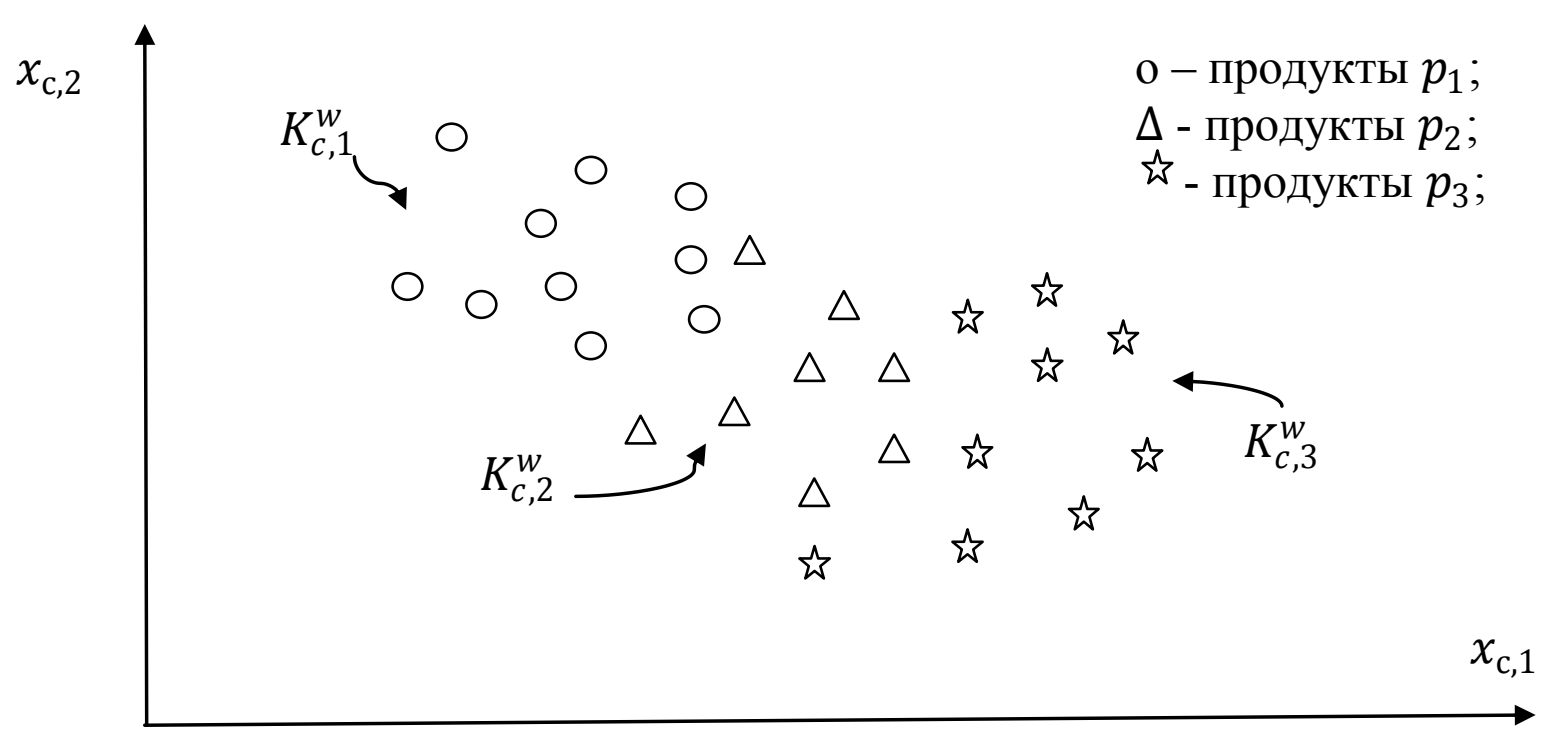

Рисунок 1 - Графическое представление классов клиентов $\left(K_{c, 1}^{w}, K_{c, 2}^{w}\right.$ и $\left.K_{c, 3}^{w}\right)$, пользующихся продуктами $p_{1}, p_{2}$ и $p_{3}$ соответственно.

Система продвижения продуктов банка может быть использована в нескольких вариантах:

1. Клиент уже работает с банком и стоит перед выбором заключения нового (или дополнительного) договора (выбора нового продукта). 
2. Клиент не работает (и не работал) с банком и выбирает продукт из некоторого множества, т.е. выбирает $p_{i} \in P, i=1,2,3, \ldots, n$.

В соответствии с этими двумя возможностями алгоритмы продвижения продуктов банка будут выглядеть следующим образом.

Алгоритм I. (Для случая, когда $c_{i_{0}} \bar{\epsilon} C$ - новый (потенциальный) клиент банка).

1. Находим $X_{c_{i_{0}}}$ (характеристики клиента $c_{i_{0}}$ );

2. Находим класс $K_{c, i_{0}}^{w}$, соответствующий $c_{i_{0}}$ и $X_{c_{i_{0}}}$;

3. Находим продукт $p_{i_{0}} \in P$, соответствующий $\mathrm{K}_{\mathrm{c}, i_{0}}^{\mathrm{w}}$ (возможно, это будет несколько продуктов);

4. В соответствии с планом развития банка (и продвижения продуктов в банке, см. табл. 3) предлагаем продукты по убыванию величин критерия $Q_{b, p_{i} \rightarrow p_{j}}$;

5. Согласовываем продукт $p_{j} \in P$ с клиентом (проводим процесс согласования); оцениваем эффективность выбора нового продукта для клиента по критерию $Q_{c, p_{i_{0}} \rightarrow p_{j}}$;

6. Заключаем (или не заключаем) договор с клиентом на продукт $p_{j} \in P$; пополняем статистику (см. табл. 1) в части w или $\overline{\mathrm{w}}$;

7. Конец работы алгоритма.

Алгоритм II. (Пусть $c_{i_{0}} \in C$ - клиент банка и он хочет заключить новый договор).

1. Характеристики $X_{c_{i_{0}}}$ известны, известен и класс $K_{c, i_{0}}^{w}$; известна динамика движения $c_{i_{0}}$ по классам:

$X_{c_{i_{0}}}(t-r \cdot \Delta t) \rightarrow X_{c_{i_{0}}}(t-(r-1) \cdot \Delta t) \rightarrow \cdots \rightarrow X_{c_{i_{0}}}(t)$,

$p_{i_{0}}(t-r \cdot \Delta t) \rightarrow p_{i_{0}}(t-(r-1) \cdot \Delta t) \rightarrow \cdots \rightarrow p_{i_{0}}(t)$,

где $t$ - текущее время;

2. В соответствии с $p_{i_{0}}(t)$ и прогнозом $p_{i_{0}}(t+\Delta t)$ находим по табл. 3 варианты развития продуктов: для клиента $c_{i_{0}}$ находим переход $p_{i_{0}} \rightarrow p_{j} ; \quad$ возможно, это будет множество продуктов; упорядочиваем эти варианты развития по убыванию значений критерия $Q_{b, p_{i_{0}} \rightarrow p_{j}}$;

3. Согласовываем продукт $p_{j} \in P$ с клиентом (осуществляется процесс согласования); оценивается эффективность выбора нового продукта для клиента $Q_{c, p_{i} \rightarrow p_{j}}$;

4. Заключается (не заключается) договор на переход к использованию клиентом продукта $p_{j} \in P$, пополняем статистику в табл. 1 (в части w или $\overline{\mathrm{w}})$;

5. Заканчивает работу алгоритм. 
Отметим некоторые особенности задачи продвижения продуктов банка и алгоритмов ее реализующих.

1. Возможны скачкообразные «движения» клиентов банка в пространстве характеристик $X_{c}$ и множества продуктов $P$ (продвижение по службе, банкротство и т.д.).

2. Из алгоритмов математической статистики выше были использованы: метод построения прогнозов, например, с использованием регрессионных моделей, и метод классификации (два алгоритма, которые могут быть реализованы в пакете программ MatLab).

3. Из алгоритмов классификации можно воспользоваться, например, алгоритмами $k$ ближайших соседей, построением деревьев классификации, построением дискриминирующих функций и др.

4. В соответствии с замечанием пп. 1, задачу прогнозирования следует решать аккуратно, возможно, только по последним 2-3 временным отсчетам (наблюдениям за значениями $p_{i_{0}}(t)$ ).

5. Возможно, при реализации алгоритмов будут востребованы и некоторые другие статистические функции пакета MatLab (регрессионного, дисперсионного анализа и т.д.).

6. Представляется интересной задача совместной обработки информации для клиентов $C_{w}$ и $C_{\bar{w}}$.

7. Измерение (оценивание) значений показателей $Q_{b, p_{i}}, Q_{c, p_{i}}, Q_{b, p_{i} \rightarrow p_{j}}$ и других может быть произведено на основе потоковых моделей соответствующих бизнес-процессов, входящих в общий процесс продвижения продуктов [3].

Рассмотрим один из подходов к разработке системы выбора параметров продуктов банка в соответствии с желаниями клиента (с учетом пожеланий клиентов) и выгодные банку (системы с «гибкими продуктами» или с «синтезированными продуктами»).

Обозначим вектор параметров договора через $\vec{\pi}=\left(\pi_{1}, \pi_{2}, \pi_{3}, \ldots, \pi_{k}\right)$. Отметим, что параметры договора - часть характеристик продукта банка из множества $X_{p_{i}}$ (множества характеристик продукта $p_{i} \in P, i=$ $1,2,3, \ldots, n)$. Этот вектор образован такими характеристиками договора, как ставки, сроки, особые условия расторжения и т.д. Пусть эффективность работы с продуктами банка (в том числе - с договорами) характеризуется критериями $\vec{Q}=\left\{Q_{1}, Q_{2}, Q_{3}, \ldots, Q_{m}\right\}$ (вектор показателей эффективности), причем, $\vec{Q}_{b}$ - критерии эффективности использования продуктов банком, $\vec{Q}_{c}$ - аналогично для клиента. 
Изобразим графически область желаемых (относительно критерия $\vec{Q}_{b}$ ) значений параметров $\vec{\pi}$ (область $\prod_{b}$, см. рис. 2 ).

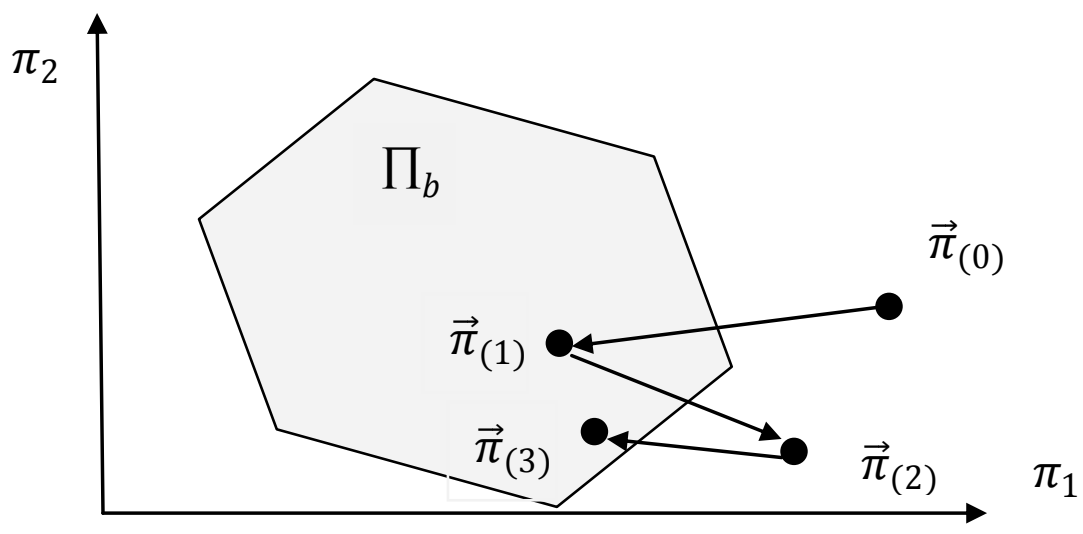

Рисунок 2 - Графическое представление области желаемых для банка значений параметров договора $\prod_{b}$ и процесса согласования параметров $\left(\vec{\pi}_{(0)} \rightarrow \vec{\pi}_{(1)} \rightarrow \vec{\pi}_{(2)} \rightarrow \vec{\pi}_{(3)}\right)$.

Рассмотрим подробнее работу алгоритма процесса согласования параметров договора по шагам.

1. Процесс согласования параметров $\vec{\pi}$ начинается с того, что клиент предлагает свои условия (желательные для него) заключения договора $\vec{\pi}_{(0)}=\vec{\pi}_{(i)}$, при $i=0, i-$ номер итерации процесса согласования; (например, это может быть набор параметров $\vec{\pi}_{(0)}=\left(t_{0}^{(0)}, T^{(0)}, r_{b}^{(0)}\right)$, где $t_{0}^{(0)}$ - время начала договора, $T^{(0)}-$ время окончания договора, $r_{b}^{(0)}-$ банковская ставка для параметров $\vec{\pi}_{(0)}$ ).

2. Находим финансовые потоки банка и характеристики этих потоков для параметров договора $\vec{\pi}_{(i)}: F_{i n}(t)$ - входной финансовый поток (от клиента банку), $F_{\text {out }}(t)$ - выходной финансовый поток (от банка клиенту), $r_{b, \text { out }}$ - ставка внешнего использования входного финансового потока банком, $r_{c, \text { out }}$ - ставка внешнего использования выходного финансового потока клиентом.

Заметим, что поток $F_{\text {in }}$ направлен от клиента к банку, а $F_{\text {out }}$, наоборот, от банка к клиенту.

3. Оцениваем значения критериев эффективности для банка. Например, оценим доход и доходность банка от потоков. Получим оценку для дохода банка (для параметров $\vec{\pi}_{(i)}$ и момента времени $T$ ):

$$
\begin{aligned}
& \Delta F_{b}(T)=N F V_{b}(T)=F_{b}^{+}(T)-F_{b}^{-}(T) ; \\
& F_{b}^{+}(T)=\sum_{t} F_{\text {in }}(t) \cdot\left(1+r_{b, \text { out }}\right)^{T-t} ; \\
& F_{b}^{-}(T)=\sum_{t} F_{\text {out }}(t)+\sum_{t} F_{\text {in }}(t) ;
\end{aligned}
$$


здесь $\sum_{t} F_{\text {out }}(t)$ - проценты по договору, которые выплатит банк клиенту, $\sum_{t} F_{i n}(t)$ - накопленный входной поток. Тогда доходность банка (для параметров $\vec{\pi}_{(i)}$ ) можно оценить по формуле:

$$
I R R_{b}=\left\{r \mid \sum_{t} F_{\text {in }}(t) \cdot(1+r)^{T-t}=\Delta F_{b}(T)\right\} .
$$

4. Моделируем поведение показателей дохода и доходности с учетом возможных рисков [3]. В частности, отклонения от оцененных значений показателей возможны из-за того, что фактические значения входного потока $F_{\text {in }}$ могут отличаться от запланированных и т.д.

5. Проверяем условие: устраивают ли значения показателей $\Delta F_{b, R}(T)$ и $I R R_{b, R}$ с учетом рисков (символ $R$ отражает учет влияния рисков на показатели эффективности) банк. Если устраивают, то оцениваем значения показателей $\Delta F_{c, R}(T)$ и $I R R_{c, R}$ для клиента и при его согласии (одобрении этих значений) заключаем договор с параметрами $\vec{\pi}_{(i)}$. Переходим на шаг 7 алгоритма.

6. Если показатели эффективности для параметров договора $\vec{\pi}_{(i)}$ не устраивают клиента или банк, то, при желании обеих сторон продолжать процесс согласования, корректируем $\vec{\pi}_{(i)}$ в соответствии с пожеланиями клиента или банка (например, увеличивая длительность договора, уменьшая банковскую ставку и т.д.) получаем (для увеличенного на единицу значения номера шага процесса согласования $i:=i+1)$ вектор параметров $\vec{\pi}_{(i)}$ и переходим на шаг 2 алгоритма. Если обе стороны решили остановить процесс согласования, то переходим на шаг 7 , так и не заключив договор.

7. Конец работы алгоритма.

Сделаем несколько замечаний.

Замечание 1. Более корректно было бы выше для показателей эффективности использовать обозначения: $\Delta F_{b}\left(T, \vec{\pi}_{(i)}\right)=N F V_{b}\left(T, \vec{\pi}_{(i)}\right)$ и $\operatorname{IRR}_{b}\left(\vec{\pi}_{(i)}\right)$, подчеркивая тем самым зависимость этих показателей от текущих значений параметров на $i$-ом шаге процесса согласования.

Замечание 2. Показатели эффективности для клиента могут быть подсчитаны в соответствии с формальными выражениями:

$$
\begin{gathered}
\Delta F_{c}(T)=N F V_{c}(T)=F_{c}^{+}(T)-F_{c}^{-}(T) ; \\
F_{c}^{+}(T)=\sum_{t} F_{\text {out }}(t) \cdot\left(1+r_{c, \text { out }}\right)^{T-t} ; \\
F_{c}^{-}(T)=\sum_{t} F_{\text {in }}(t) \cdot\left(1+r_{c, \text { alt }}\right)^{T-t}, \\
I R R_{\mathrm{c}}=\left\{r \mid \sum_{t} F_{\text {in }}(t) \cdot(1+r)^{T-t}=\Delta F_{\mathrm{c}}(T)\right\},
\end{gathered}
$$

где $r_{c, a l t}$ - альтернативная ставка для клиента.

Замечание 3. Методику согласования параметров договора можно использовать в двух аспектах. Либо так как это делается в настоящее время (согласовать с клиентом банка один из вариантов договора из фиксированного множества параметров), либо синтезировать 
согласованный вектор параметров в соответствии с рассмотренным выше алгоритмом.

Показатели эффективности с учетом рисков можно оценить, если воспользоваться процедурой моделирования [3]. Так, например, если в результате моделирования значений показателя $I R R_{b}$ получилась некоторая эмпирическая функция распределения вероятностей для этого показателя $F\left(I R R_{b}\right)$, то в качестве показателя эффективности с учетом рисков $I R R_{b, R}$ можно использовать следующие значения: $I R R_{b, R}=I R R_{b}-$ $\arg \left(\left(\min \left(F\left(I R R_{b}\right)\right) \mid F\left(I R R_{b}\right)>0\right)\right) ; \quad I R R_{b, R}=I R R_{b}-\operatorname{VaR}\left(q, F\left(I R R_{b}\right)\right)$. Здесь $\arg \left(\left(\min \left(F\left(I R R_{b}\right)\right) \mid F\left(I R R_{b}\right)>0\right)\right)$ - это то значение показателя $I R R_{b}$, которому соответствует наименьшее значение функции $I R R_{b}$, или наименьшее из полученных при моделировании значение $I R R_{b}$, $\operatorname{VaR}\left(q, F\left(I R R_{b}\right)\right)-\operatorname{VaR}$-показатель риска.

\section{Литература}

1. Чистов Д.В. Банковские информационные системы и технологии. Москва: Финансы и статистика, 2005. - 384 с.

2. Банковские информационные системы: Учебник/ Под ред. проф. В.В. Дика. - М.: Маркет ДС, 2010. -816 с.

3. Наумов А.А. Теоретические и прикладные вопросы моделирования бизнес-процессов. Модели, алгоритмы, программы. - LAP LAMBERT Academic Publishing, 2012. - 464 c. 